\title{
Revealed preferences towards the appraisal of orphan drugs in Poland - multi criteria decision analysis
}

\author{
Katarzyna Kolasa ${ }^{1 *}$ (D), Krzysztof Miroslaw Zwolinski ${ }^{2}$, Vladimir $Z^{2}{ }^{3}$, Zoltán Kaló ${ }^{4,6}$ and Tadeusz Lewandowski ${ }^{5}$
}

\begin{abstract}
Background: A Multi Criteria Decision Analysis (MCDA) technique was adopted to reveal the preferences of the Appraisal Body of the Polish HTA agency towards orphan drugs (OMPs).

Results: There were 34 positive and 23 negative HTA recommendations out of 54 distinctive drug-indication pairs. The MCDA matrix consisted of 13 criteria, seven of which made the most impact on the HTA process. Appraisal of clinical evidence, cost of therapy, and safety considerations were the main contributors to the HTA guidance, whilst advancement of technology and manufacturing costs made the least impact.

Conclusions: MCDA can be regarded as a valuable tool for revealing decision makers' preferences in the healthcare sector. Given that only roughly half of all criteria included in the MCDA matrix were deemed to make an impact on the HTA process, there is certainly some room for improvement with respect to the adaptation of a new approach towards the value assessment of OMPs in Poland.
\end{abstract}

Keywords: Revealed preferences, Multi criteria decision making, Orphan drugs, Health technology assessment, Poland, Economic and non-economic decision making criteria

\section{Background}

There are up to 36 million individuals suffering from as many as 5000-8000 different types of rare diseases in the EU. Although many of them significantly impact life expectancy, available data suggests that there is still considerable number of patients that remains without the treatment [1].

Given the growing challenges with limited budgets being spent on innovative orphan medicine products (OMPs), more transparent and objective pricing \& reimbursement (P\&R) decision-making process is sought. Among new initiatives, the Transparent Value Framework (TVF) developed by the Working Group on Mechanism for Coordinated Access to OMPs (MoCA) could be pointed out [2]. The TVF directs special attention towards the inclusion of non-economic criteria, such as unmet medical needs and availability of alternative treatment options in the $P \& R$ process of OMPs. It attempts to evaluate feasibility of the inclusion of a broad scope of decision-making criteria in

\footnotetext{
* Correspondence: kkolasa@kozminski.edu.pl

${ }^{1}$ Department of Health Economics and Healthcare Management, Kozminski

University, Warsaw, Poland

Full list of author information is available at the end of the article
}

the efforts towards more transparent and equitable assessment of the value of OMPs.

Despite limited number of examples of specific P\&R frameworks being established for the evaluation of OMPs, majority of jurisdictions have not separated reimbursement processes for OMPs and non-OMPs [3-5]. With that, it is interesting to ask what kind of criteria is utilized for the assessment of OMPs in the P\&R system without any special arrangements for the evaluation of treatments for rare diseases. It is particularly valuable to provide a relevant insight into whether the broader set of criteria as envisaged by the MoCA project is at least implicitly taken into consideration in the appraisal processes of OMPs.

Central and Eastern European (CEE) settings can serve as an interesting research field for that purpose. On the one hand, criteria of clinical and cost effectiveness analysis alongside budget impact analysis are commonly established in the format of standard HTA, and there are some instances where the fixed cost effectiveness threshold is explicitly set amongst P\&R rules for both orphan and non-orphan drug technologies $[6,7]$. On the other hand, CEE countries lag behind the rest of the EU with regard 
to accessibility to treatment for patients with rare diseases. For instance, it was revealed that only in 22 out of registered 61 , and 28 out of registered 72 OMPs were available in Bulgaria and Latvia respectively [8-10]. According to the EURORDIS published study, as many as $90 \%$ of 60 OMPs were covered by public funds in the Netherlands, whilst only $30 \%$ were covered in Romania [10].

The Polish jurisdiction was chosen for the purpose of this study. It is the biggest country in the CEE Region with a well-established HTA process. The proceedings from the P\&R negotiations are not available in the public domain. In contrast to other countries in the CEE Region, the Polish HTA Agency does, however, publish its recommendations.

The objective of our study was to reveal the value criteria appraised in the HTA appraisal process in Poland. The Polish Appraisal Body consists of the President of the HTA agency and ten members of the Appraisal Committee. Among them, there are representatives of the Ministry of Health, the National Health Fund, regulatory body, and patients' ombudsman [11]. The HTA recommendations are not mandatory in Poland. The pricing negotiations are conducted at the discretion of the Ministry of Health.

The Multi Criteria Decision Analysis (MCDA) framework was chosen for the purpose of the study. There is an increasing interest in the MCDA's implementation into the P\&R process as it allows for a trade-off between different decision-making criteria [12]. In the particular case of our research, it provides an opportunity to reveal the preferences towards value criteria, and to assign weights to each of them representing their importance in the HTA appraisal process. It was hypothesized that findings from our research can help elicit recommendations on how to achieve more transparency in the pricing and reimbursement process for OMPs.

\section{Methods}

The analysis was divided into five steps following the ISPOR checklist designed for MCDA studies [15]:

1. Description of the decision problem,

2. Selecting and defining criteria,

3. Measuring performance,

4. Scoring and weighting criteria,

5. Dealing with uncertainty.

The MCDA analysis was performed utilizing validated ZRx MCDA tool [13].

\section{Description of the decision problem}

The decision problem was set to reveal the OMPs' value criteria appraised in the HTA process between January 2008 and June 2015 by the Polish HTA Agency (AHTAPoL).

\section{MCDA matrix development}

An MCDA matrix was conceived based on the set of value attributes of the OMPs identified in the systematic review of the literature published elsewhere [14]. The pilot review of 10 HTA recommendations was conducted to reveal potential additional criteria utilized in the appraisal process. Following ISPOR's guidelines, the attributes were chosen with respect to certain principles such as completeness, non-redundancy, non-overlap, and preferential independence [15].

\section{Measuring performance \\ Review of HTA recommendations}

A database of HTA recommendations issued with respect to OMPs was established. Each indication for the same active substance was considered separately and treated as a distinctive case (drug-indication pair). HTA recommendations issued by both the Appraisal Body and, if available, by the President of the Polish HTA agency, were used for the purpose of the study (HTA outcomes). The categorization of HTA outcomes into negative and positive ones was carried out, and reasons for each recommendation were elicited [16].

\section{MCDA matrix population}

Two independent reviewers conducted the review of HTA recommendations to identify which MCDA criteria were considered in the HTA process for each drug-indication pair separately. Any disagreement was resolved by consensus. An MCDA matrix was established in the table format known as "matrix performance" [17]. It was populated with the data from each HTA recommendations so that one of three different numbers could be assigned to each drug-indication pair for each MCDA criteria separately: 1full deliberation, in case a given criteria was utilized to justify the HTA recommendation, 0.5- partial deliberation, in case a given criteria was only mentioned in the HTA report without being used for justification of the HTA recommendation, and 0- lack of deliberation.

\section{Scoring and weighting criteria MCDA analysis}

Multiple Attribute Value Theory (MAVT) was adopted to reveal the preferences of the HTA Appraisal Body. This is a methodology designed to address complex decision making problems involving multiple attributes. In principle, it compares different alternatives against each other using a number of distinctive criterions. A simple linear additive model (SLAM) and analytic hierarchy process (AHP) were adopted for the purpose of this study [18]. The SLAM method evaluates alternatives against a chosen set of criteria taking into consideration both preferences for each criteria (weights) and performance of each alternative against those criteria. We utilized SLAM method to compare the frequency of 
deliberation of each criterion (model's alternatives) in HTA process across all studied cases (alternatives' performance). It was assumed that each drug indication pair contributed equally to the analysis (model's weights); hence, equal weights were assigned to each HTA recommendation. In contrast to SLAM, AHP analysis is based on a special matrix that allows measuring the intensity of importance of each MCDA criteria in a pair-wise comparison. In that study, the relative importance of each criterion against another was ranked on the scale that corresponds to the number of drug indications pairs used.

A normalized matrix allowed assigning weight to each criteriion based on its importance in the HTA appraisal process as well. The sum of the weights equalled $100 \%$. For both SLAM and AHP, the frequency of the deliberation of each MCDA criterion between groups of positive and negative HTA outcomes was compared. In cases of a lack of significant differences, an MCDA analysis was conducted jointly for both groups. Otherwise, a separate MCDA processes was planned. Further methodological details regarding MCDA analysis applied in the study are presented in the Additional file 1.

\section{Dealing with uncertainty \\ Sensitivity analysis}

In the sensitivity analysis, both the Variable Interdependent Parameters (VIP) and maximal regret methods were utilized. For the purpose of this particular study, we assumed a threshold of 0.5 for both VIP minimum value and maximal regret to assess the importance of the given criteria. Further details are available in the supplementary materials.

\section{Results}

Data

The MCDA matrix consisted of 13 criteria, ten of which were imported from the other study [14]. Additionally, three criteria were added as a result of the review of ten randomly selected HTA recommendations. It was therapy costs, the impact of other HTA guidelines on the Polish HTA appraisal, and the results of rationalization analysis whose objective is to identify financial sourcing for the technology in question. The definition of the remaining ten criteria is presented elsewhere [14].

In total, 57 distinctive drug-indication pairs were included in the dataset. Based on the review of HTA recommendations, 34 positive and 23 negative HTA outcomes were distinguished (Table 1).

In 19 out of 23 negative HTA outcomes, insufficient clinical evidence was discussed (Table 1). Economic concerns were mentioned on seven occasions, and that was the sole reason for rejection in three cases. The unfavorable results of a cost effectiveness analysis were mentioned most frequently amongst the non-clinical considerations.
In 15 out of 34 positive HTA outcomes, no restrictions were mentioned (Table 1). Among the remaining ones, the recommendation for use limited to certain subgroups of patients was the most often raised suggestion. The Appraisal Body required lowering the cost of therapy in nine cases.

\section{MCDA analysis}

The deliberation of MCDA criteria between drug indication pairs with positive and negative HTA outcomes was compared. Apart from the consideration of safety aspects and the results of rationalization analysis, no significant differences were observed between both groups (Table 2). Consequently, MCDA analysis was conducted jointly for the total dataset.

The results of the SLAM analysis are presented in Table 2. They indicate that clinical evidence was the most frequently deliberated criteria in the Polish HTA Appraisal process (Table 2). This was followed by the notion of therapeutic costs, consideration of safety aspects, and the availability of alternative treatment options. The data regarding manufacturing technology, advancement in technology, and the results of the rationalization analysis were deemed to be the least important.

The AHP results are presented in Table 3 . The matrix of pairwise comparison revealed that the value of the clinical evidence outweighed all other attributes in the HTA process. Its importance ranged from being 1.3 to 55 times higher than safety and manufacturing technology aspects respectively. With a weight of $17 \%$, clinical evidence had the greatest impact on the outcome of the HTA process (Table 4), whilst therapeutic costs and safety aspects, with weights of $14 \%$ and $13 \%$ respectively, contributed significantly to the HTA outcome as well (Table 4). The pairwise comparison revealed that both criteria were less important than clinical evidence but more important than all the remaining ones. The significance of their value against other attributes ranged from 1.5 to 46 times compared to CEA results and manufacturing costs respectively. With a weight of $10 \%$, availability of therapeutic alternative was the fourth most impactful contributor to the HTA recommendation process (Table 4). Although it was less important than clinical evidence, safety, and therapeutic costs, according to the pairwise comparison, it was still more influential than nine others. The importance of therapeutic alternative was valued at almost twice as much as the one for CEA and BIA, and more than 30 times greater than the manufacturing technology (Table 3). As far as CEA and BIA results are concerned, they almost equally contributed to the HTA recommendation process with a weight of $9 \%$ each (Table 4). Although they informed the HTA process to a lesser extent than the four above discussed criteria, both CEA and BIA results were more important 
Table 1 HTA outcomes for OMPs issued by the Polish HTA agency between

\begin{tabular}{|c|c|c|c|c|c|}
\hline Entry & Brand name (Active substance) & Indication & $\begin{array}{l}\text { HTA } \\
\text { outcome }\end{array}$ & Data source & $\begin{array}{l}\text { Reason for HTA } \\
\text { recommendation }\end{array}$ \\
\hline 1 & Adcetris (brentuximab vedotin) & $\begin{array}{l}\text { Lymphoma CD30+: Hodgkin } \\
\text { Disease (C81), Lymphoma, Non- } \\
\text { Hodgkin (C84.5) }\end{array}$ & Negative & $\begin{array}{l}\text { Rekomendacja prezesa AOTM } \\
\text { nr 96/2013 }\end{array}$ & $\begin{array}{l}\text { Clinical reasons: insufficient } \\
\text { evidence for use (poor quality } \\
\text { data). Economic reasons: } \\
\text { insufficient justification of the } \\
\text { treatments cost in relation to its } \\
\text { benefit }\end{array}$ \\
\hline 2 & Adempas (riociguat) & $\begin{array}{l}\text { Chronic Thromboembolic } \\
\text { Pulmonary Hypertension } \\
\text { (CTEPH) (ICD-10 I27, I27.0 and/ } \\
\text { or I26) }\end{array}$ & Positive & $\begin{array}{l}\text { Rekomendacja prezesa AOTM } \\
\text { nr 261/2014 }\end{array}$ & $\begin{array}{l}\text { Minor restrictions: use at lower } \\
\text { price }\end{array}$ \\
\hline 3 & Arzerra (ofatumumab) & $\begin{array}{l}\text { Chronic Lymphocytic Leukemia } \\
\text { in patients who are refractory to } \\
\text { fludarabine and alemtuzumab }\end{array}$ & Negative & $\begin{array}{l}\text { Rekomendacja prezesa AOTM } \\
\text { nr 5/2012 }\end{array}$ & $\begin{array}{l}\text { Clinical reasons: insufficient } \\
\text { evidence for use (poor quality } \\
\text { data). Economic reasons: } \\
\text { unacceptable budget impact, } \\
\text { insufficient justification of the } \\
\text { treatments cost in relation to its } \\
\text { benefit }\end{array}$ \\
\hline 4 & Atriance (Nelarabine) & $\begin{array}{l}\text { treatment of patients with T-cell } \\
\text { acute lymphoblastic leukaemia } \\
\text { (T-ALL) and T-cell lymphoblastic }\end{array}$ & Positive & $\begin{array}{l}\text { Stanowisko Rady } \\
\text { Konsultacyjnej nr 13/04/2009 }\end{array}$ & $\begin{array}{l}\text { Minor restrictions: use at lower } \\
\text { price }\end{array}$ \\
\hline
\end{tabular}
(T-ALL) and T-cell lymphoblastic Konsultacyjnej nr 13/04/2009 price lymphoma (T-LBL) whose disease has not responded to or has relapsed following treatment with at least two chemotherapy regimens, eligible for a bone marrow transplant

5 Bramitob (tobramycin)

$6 \quad$ Cometriq (cabozantinib) treatment of Pseudomonas aeruginosa lung infection in cystic fibrosis (ICD-10 E84)

Thyroid Neoplasms (ICD-10 C73)
Negative Rekomendacja prezesa AOTM nr 83/2013

Negative

Rekomendacja prezesa AOTM nr 51/2015
Mucopolysaccharidosis type II, MPS II (Hunter syndrome) long-term treatment

Idiopathic Pulmonary Fibrosis (ICD-10 J 84.1)

Treatment of acute lymphoblastic leukaemia (ALL) in paediatric patients who have relapsed or are refractory after receiving at least two prior regimens and where there is no other treatment option anticipated to result in a durable response, in patients eligible for a hemapoietic stem cell transplant

Treatment of chronic iron overload
Positive Rekomendacja prezesa AOTM nr 6/2010, Stanowisko Rady Konsultacyjnej nr 9/3/2010

Negative Komunikat na stronie www AOTM

Negative Rekomendacja prezesa AOTM nr 79/2013

Positive Rekomendacja prezesa AOTM nr 127/2012
Economic reasons: insufficient justification of the treatments cost in relation to its benefit

Clinical reasons: insufficient evidence for use (inappropriate comparator or poor quality data), poor safety. Economic reasons: poor economic data, insufficient justification of the treatments cost in relation to its benefit

inor restriction: monitoring required

Clinical reasons: insufficient evidence for use (poor quality data)

Clinical reasons: insufficient evidence for use (poor quality data), poor safety

Major restriction: used restricted to specific subpopulation
Positive Rekomendacja prezesa AOTM Minor restriction: monitoring nr 68/2012 required 
Table 1 HTA outcomes for OMPs issued by the Polish HTA agency between (Continued)

\begin{tabular}{|c|c|c|c|c|c|}
\hline Entry & Brand name (Active substance) & Indication & $\begin{array}{l}\text { HTA } \\
\text { outcome }\end{array}$ & Data source & $\begin{array}{l}\text { Reason for HTA } \\
\text { recommendation }\end{array}$ \\
\hline 12 & Fabrazyme (Agalsidase beta) & $\begin{array}{l}\text { Fabry disease (alpha- } \\
\text { galactosidase A deficiency) - } \\
\text { long-term replace therapy }\end{array}$ & Negative & $\begin{array}{l}\text { Stanowisko Rady } \\
\text { Konsultacyjnej nr 20/06/2009 }\end{array}$ & $\begin{array}{l}\text { Clinical reasons insufficient } \\
\text { evidence for use (inappropriate } \\
\text { comparator or poor quality } \\
\text { data), poor safety. Economic } \\
\text { reasons: insufficient justification } \\
\text { of the treatments cost in } \\
\text { relation to its benefit }\end{array}$ \\
\hline 13 & Firazyr (icatibant) & $\begin{array}{l}\text { Treatment of acute attacks of } \\
\text { hereditary angioedema (HAE) in } \\
\text { adults (with C1-esterase- } \\
\text { inhibitor deficiency) }\end{array}$ & Negative & $\begin{array}{l}\text { Rekomendacja prezesa AOTM } \\
\text { nr 22/2015 }\end{array}$ & $\begin{array}{l}\text { Economic reasons: insufficient } \\
\text { justification of the treatments } \\
\text { cost in relation to its benefit }\end{array}$ \\
\hline 14 & Gazyvaro (obinutuzumab) & $\begin{array}{l}\text { Chronic lymphocytic leukaemia } \\
\text { (CLL) (ICD-10: C.91.1) }\end{array}$ & Negative & $\begin{array}{l}\text { Rekomendacja prezesa AOTM } \\
\text { nr 60/2015 }\end{array}$ & $\begin{array}{l}\text { Clinical reasons: insufficient } \\
\text { evidence for use (inappropriate } \\
\text { comparator or poor quality } \\
\text { data), poor safety. Economic } \\
\text { reasons: poor economic data, } \\
\text { insufficient justification of the } \\
\text { treatments cost in relation to its } \\
\text { benefit }\end{array}$ \\
\hline 15 & Glivec (Imatinib) & $\begin{array}{l}\text { Myelodysplastic/ } \\
\text { myeloproliferative diseases } \\
\text { (MDS/MPD) }\end{array}$ & Positive & $\begin{array}{l}\text { Rekomendacja prezesa AOTM } \\
\text { nr 7/2011 }\end{array}$ & Unrestricted \\
\hline 16 & Glivec (Imatinib) & $\begin{array}{l}\text { Dermatofibrosarcoma } \\
\text { protuberans (DFSP) }\end{array}$ & Positive & $\begin{array}{l}\text { Rekomendacja prezesa AOTM } \\
\text { nr } 5 / 2011\end{array}$ & Unrestricted \\
\hline 17 & Glivec (Imatinib) & $\begin{array}{l}\text { Malignant gastrointestinal } \\
\text { stromal tumors (GIST) }\end{array}$ & Positive & $\begin{array}{l}\text { Komunikat wraz z } \\
\text { uzasadnieniem na stronie } \\
\text { AHTAPol }\end{array}$ & Unrestricted \\
\hline 18 & Glivec (Imatinib) & $\begin{array}{l}\text { Philadelphia chromosome } \\
\text { positive chronic myeloid } \\
\text { leukemia (ALL Ph+) }\end{array}$ & Positive & $\begin{array}{l}\text { Rekomendacja prezesa AOTM } \\
\text { nr 6/2011 }\end{array}$ & Unrestricted \\
\hline 19 & Increlex (Mecasermin) & $\begin{array}{l}\text { Insulin-like growth factor } \\
\text { deficiency -IGF-1 (Laron } \\
\text { Syndrome) - long-term } \\
\text { treatment }\end{array}$ & Positive & $\begin{array}{l}\text { Stanowisko Rady } \\
\text { Konsultacyjnej nr 43/12/2009 }\end{array}$ & $\begin{array}{l}\text { Major restriction: resubmission } \\
\text { required after certain time }\end{array}$ \\
\hline 20 & Jakavi (ruxolitinib) & $\begin{array}{l}\text { primary myelofibrosis (also } \\
\text { known as chronic idiopathic } \\
\text { myelofibrosis), post- } \\
\text { polycythaemia-vera myelofibro- } \\
\text { sis or post-essential- } \\
\text { thrombocythaemia } \\
\text { myelofibrosis }\end{array}$ & Positive & $\begin{array}{l}\text { Rekomendacja prezesa AOTM } \\
\text { nr 120/2014 }\end{array}$ & Unrestricted \\
\hline 21 & Kalydeco (ivacaftor) & Cystic fibrosis (CF) (ICD-10 E84) & Negative & $\begin{array}{l}\text { Rekomendacja prezesa AOTM } \\
\text { nr 54/2015 }\end{array}$ & $\begin{array}{l}\text { Clinical reasons: insufficient } \\
\text { evidence for use (poor quality } \\
\text { data). Economic reasons: } \\
\text { insufficient justification of the } \\
\text { treatments cost in relation to } \\
\text { its benefit }\end{array}$ \\
\hline 22 & Kuvan (sapropterin) & $\begin{array}{l}\text { Hyperphenylalaninemia (HPA) in } \\
\text { patients with } \\
\text { tetrahydrobiopterin (BH4) } \\
\text { deficiency }\end{array}$ & Positive & $\begin{array}{l}\text { Rekomendacja prezesa AOTM } \\
\text { nr 55/2011 }\end{array}$ & $\begin{array}{l}\text { Major restriction: resubmission } \\
\text { required after certain time }\end{array}$ \\
\hline 23 & Mabthera (rituximab) & Non-Hodgkin's lymphoma (NHL) & Positive & $\begin{array}{l}\text { Rekomendacja prezesa AOTM } \\
\mathrm{nr} 7 / 2012\end{array}$ & $\begin{array}{l}\text { Major restriction: used restricted } \\
\text { to specific subpopulation }\end{array}$ \\
\hline 24 & Mabthera (rituximab) & $\begin{array}{l}\text { Non-Hodgkin's lymphoma } \\
\text { classified to code ICD-10 C84 }\end{array}$ & Negative & $\begin{array}{l}\text { Rekomendacja prezesa AOTM } \\
\text { nr 24/2012 }\end{array}$ & $\begin{array}{l}\text { Clinical reason: insufficient } \\
\text { evidence for use } \\
\text { (inappropriate comparator or } \\
\text { poor quality data) }\end{array}$ \\
\hline 25 & Mabthera (rituximab) & $\begin{array}{l}\text { Code ICD-10 C85 (Other and } \\
\text { unspecified types of non- } \\
\text { Hodgkin lymphoma) }\end{array}$ & Positive & $\begin{array}{l}\text { Rekomendacja prezesa AOTM } \\
\mathrm{nr} 25 / 2012\end{array}$ & Unrestricted \\
\hline
\end{tabular}


Table 1 HTA outcomes for OMPs issued by the Polish HTA agency between (Continued)

\begin{tabular}{lll}
\hline Entry & Brand name (Active substance) & Indication \\
\hline $26 \quad$ Mabthera (rituximab) & $\begin{array}{l}\text { Hodgkin Lymphoma (Hodgkin } \\
\text { disease-HD) } \\
\text { Osteosarcoma (ICD-10 C40-41) }\end{array}$ \\
& \\
& \\
& \\
& \\
& In combination with \\
& granulocyte-colony-stimulating \\
& factor to enhance mobilisation \\
& of haematopoietic stem cells to \\
& the peripheral blood for collec- \\
& tion and subsequent autologous \\
& transplantation in patients with \\
& lymphoma and multiple mye- \\
& loma whose cells mobilise \\
poorly (ICD-10: C81-85, C90) & \\
&
\end{tabular}

Myozyme (alglucosidase alfa)

30/ Nexavar (Sorafenib)

31

32 Nexavar (Sorafenib)

33 Nplate (Romiplostim)

34 Opsumit (macitentan)

35 Revatio (Sildenafil)

36 Revlimid (Lenalidomide)

37 Revlimid (Lenalidomide)

38 Signifor (pasireotide)

39
Pompe disease (acid-aglucosidase deficiency) (ICD-10 E74.0)

Renal cell carcinoma (RCC)

Hepatocellular carcinoma (HCC)

Chronic immune (idiopathic) thrombocytopenic purpura (ITP)

long-term treatment of pulmonary arterial hypertension $(\mathrm{PAH})$ in combination (ICD-10 127, 127.0)

Pulmonary arterial hypertension (PAH)

Myelodysplastic/

Myeloproliferative syndrome (MM/S)

(off-label indication)

Myelodysplastic/

Myeloproliferative syndrome (MM/S)

Cushing's disease for whom surgery is not an option or for whom surgery has failed (ICD10 E 24.0)

Acromegaly

HTA Data source

outcome

Positive

Rekomendacja prezesa AOTM nr 19/2012

Reason for HTA

recommendation

Negative Rekomendacja prezesa AOTM nr 78/2013

Negative Rekomendacja prezesa AOTM nr 182/2013

Negative Rekomendacja prezesa AOTM nr 8/2013

Clinical reason: insufficient evidence for use (poor quality data). Economic reasons: insufficient justification of the treatments cost in relation to its benefit

Negative Rekomendacja prezesa AOTM nr 48/2009, Stanowisko Rady Konsultacyjnej $\mathrm{nr}$ 27/10/26/ 2009, and Uchwała Rady Konsultacyjnej nr 22/07/2008

Clinical reasons: insufficient evidence for use (poor quality data), poor efficacy. Economic reasons: insufficient justification of the treatments cost in relation to its benefit

Positive Rekomendacja prezesa AOTM nr 26/2010

Major restriction: used restricted to specific subpopulation

Positive Rekomendacja prezesa AOTM nr 13/2010 to specific subpopulation

Negative Rekomendacja prezesa AOTM nr 23/2015

Positive

Uchwała Rady Konsultacyjnej nr 1/01/2008

Positive Rekomendacja prezesa AOTM nr 83/2011

Clinical reasons: insufficient evidence for use (inappropriate comparator or poor quality data). Economic reasons: unacceptable budget impact

Unrestricted

Off-label indication. Major restriction: used restricted to specific subpopulation. Minor restriction: use at lower price

Positive Rekomendacja prezesa AOTM Major restriction: used restricted nr $11 / 2012$ to specific subpopulation. Minor restriction: use at lower price

Positive Rekomendacja prezesa AOTM Minor restriction: use at lower nr 99/2013 price

Negative Rekomendacja prezesa AOTM nr 4/2011
Clinical reasons: insufficient evidence for use (inappropriate comparator or poor quality data), poor efficacy. Economic reasons: insufficient justification of the treatments cost in relation to its benefit 
Table 1 HTA outcomes for OMPs issued by the Polish HTA agency between (Continued)

\begin{tabular}{|c|c|c|c|c|c|}
\hline Entry & Brand name (Active substance) & Indication & $\begin{array}{l}\text { HTA } \\
\text { outcome }\end{array}$ & Data source & $\begin{array}{l}\text { Reason for HTA } \\
\text { recommendation }\end{array}$ \\
\hline 40 & Sprycel (Dasatinib) & Chronic myeloid leukemia (CML) & Positive & $\begin{array}{l}\text { Uchwała Rady Konsultacyjnej } \\
\text { nr 23/07/2008 }\end{array}$ & $\begin{array}{l}\text { Major restriction: use only as } \\
\text { second or subsequent line } \\
\text { treatment }\end{array}$ \\
\hline 41 & Sprycel (dasatinib) & $\begin{array}{l}\text { Indication clacisified to codes: } \\
\text { ICD10:C96.2, within non- } \\
\text { standard chemiotherapy } \\
\text { programme }\end{array}$ & Negative & $\begin{array}{l}\text { Rekomendacja prezesa AOTM } \\
\text { nr 66/2014 }\end{array}$ & Clinical reasons: poor efficacy \\
\hline 42 & Sutent (sunitinib) & $\begin{array}{l}\text { unresectable or metastatic } \\
\text { malignant gastrointestinal } \\
\text { stromal tumors (GIST) in adults } \\
\text { with disease progression }\end{array}$ & Positive & $\begin{array}{l}\text { Rekomendacja prezesa AOTM } \\
\text { nr 20/2012 }\end{array}$ & Unrestricted \\
\hline 43 & Tasigna (Nilotinib) & $\begin{array}{l}\text { Chronic myeloid leukemia (CML) } \\
\text { with resistance or intolerance to } \\
\text { prior therapy }\end{array}$ & Positive & $\begin{array}{l}\text { Uchwała Rady Konsultacyjnej } \\
\text { nr 53/15/2008 }\end{array}$ & $\begin{array}{l}\text { Major restriction: use only if } \\
\text { intolerant to other treatment. } \\
\text { Minor restriction: use at } \\
\text { lower price }\end{array}$ \\
\hline 44 & $\begin{array}{l}\text { Thalidomide Celgene } \\
\text { (thalidomide) }\end{array}$ & $\begin{array}{l}\text { In combination with melphalan } \\
\text { and prednisone as first-line } \\
\text { treatment of patients with un- } \\
\text { treated multiple myeloma aged } \\
\geq 65 \text { years or ineligible for high- } \\
\text { dose chemotherapy }\end{array}$ & Positive & $\begin{array}{l}\text { Rekomendacja prezesa AOTM } \\
\text { nr 106/2012 }\end{array}$ & $\begin{array}{l}\text { Minor restriction: use at lower } \\
\text { price }\end{array}$ \\
\hline 45 & Torisel (Temsirolimus) & Renal cell carcinoma (RCC) & Negative & $\begin{array}{l}\text { Rekomendacja prezesa AOTM } \\
\text { nr } 47 / 2009 \text {. } \\
\text { Stanowisko Rady } \\
\text { Konsultacyjnej nr 26/10/26/ } \\
2009\end{array}$ & $\begin{array}{l}\text { Clinical reasons: insufficient } \\
\text { evidence for use (inappropriate } \\
\text { comparator or poor quality data). } \\
\text { Economic reasons: insufficient } \\
\text { justification of the treatments } \\
\text { cost in relation to its benefit }\end{array}$ \\
\hline 46 & Torisel (temsirolimus) & $\begin{array}{l}\text { treatment of adult patients with } \\
\text { advanced renal-cell carcinoma } \\
\text { (RCC) with unfavorable prognos- } \\
\text { tic (ICD-10: C64) (RCC-up) }\end{array}$ & Negative & $\begin{array}{l}\text { Rekomendacja prezesa AOTM } \\
\text { nr 58/2013 }\end{array}$ & $\begin{array}{l}\text { Clinical reasons: insufficient } \\
\text { evidence for use (inappropriate } \\
\text { comparator or poor quality } \\
\text { data), poor safety. Economic } \\
\text { reasons: insufficient justification } \\
\text { of the treatments cost in } \\
\text { relation to its benefit }\end{array}$ \\
\hline 47 & Tracleer (Bosentan) & $\begin{array}{l}\text { Pulmonary arterial hypertension } \\
\text { (PAH) }\end{array}$ & Positive & $\begin{array}{l}\text { Uchwała Rady Konsultacyjnej } \\
\text { nr 1/01/2008 }\end{array}$ & $\begin{array}{l}\text { Major restrictions: used } \\
\text { restricted to specific } \\
\text { subpopulation, use only as } \\
\text { second or subsequent line } \\
\text { treatment. Minor restriction: } \\
\text { monitoring required. }\end{array}$ \\
\hline 48 & Trisenox (arsenic trioxide) & $\begin{array}{l}\text { for induction of remission and } \\
\text { consolidation in adult patients } \\
\text { with pro-Myelotic Leucaemia } \\
\text { (APL)/ Retinoic-Acid receptor- } \\
\text { alpha PML/RAR alpha }\end{array}$ & Negative & $\begin{array}{l}\text { Rekomendacja prezesa AOTM } \\
\text { nr } 6 / 2012\end{array}$ & $\begin{array}{l}\text { Clinical reason: insufficient } \\
\text { evidence for use (poor quality } \\
\text { data). Economic reason: insufficient } \\
\text { justification of the treatments cost } \\
\text { in relation to its benefit }\end{array}$ \\
\hline 49 & Ventavis (lloprost) & $\begin{array}{l}\text { Pulmonary arterial hypertension } \\
\text { (PAH) }\end{array}$ & Positive & $\begin{array}{l}\text { Uchwała Rady Konsultacyjnej } \\
\text { nr 1/01/2008 }\end{array}$ & $\begin{array}{l}\text { Major restrictions: used } \\
\text { restricted to specific } \\
\text { subpopulation, use only as } \\
\text { second or subsequent line } \\
\text { treatment. Minor restriction: } \\
\text { monitoring required. }\end{array}$ \\
\hline 50 & Vidaza (Azacitidine) & $\begin{array}{l}\text { Acute myelogenous leukemia } \\
\text { (AML) }\end{array}$ & Positive & $\begin{array}{l}\text { Rekomendacja prezesa AOTM } \\
\text { nr 18/2011 }\end{array}$ & Unrestricted \\
\hline 51 & Volibris (Ambrisentan) & $\begin{array}{l}\text { Pulmonary arterial hypertension } \\
\text { (PAH) (ICD-10 I27, 127.0) }\end{array}$ & Positive & $\begin{array}{l}\text { Rekomendacja prezesa AOTM } \\
\text { nr 29/2010 }\end{array}$ & Unrestricted \\
\hline 52 & Votubia (everolimus) & $\begin{array}{l}\text { Treatment of patients with } \\
\text { subependymal giant cell }\end{array}$ & Positive & $\begin{array}{l}\text { Rekomendacja prezesa AOTM } \\
\text { nr } 81 / 2014\end{array}$ & $\begin{array}{l}\text { Minor restriction: use at lower } \\
\text { price }\end{array}$ \\
\hline
\end{tabular}
astrocytoma (SEGA) associated with tuberous sclerosis complex (TSC) who require therapeutic intervention but 
Table 1 HTA outcomes for OMPs issued by the Polish HTA agency between (Continued)

\begin{tabular}{|c|c|c|c|c|c|}
\hline Entry & Brand name (Active substance) & Indication & $\begin{array}{l}\text { HTA } \\
\text { outcome }\end{array}$ & Data source & $\begin{array}{l}\text { Reason for HTA } \\
\text { recommendation }\end{array}$ \\
\hline & & $\begin{array}{l}\text { are not amenable to surgery } \\
\text { (ICD-10 Q85.1) }\end{array}$ & & & \\
\hline 53 & Vpriv (velaglucerase alfa) & Gaucher disease (ICD-10: E-75) & Positive & $\begin{array}{l}\text { Rekomendacja prezesa AOTM } \\
\text { nr 120/2013 }\end{array}$ & $\begin{array}{l}\text { Minor restriction: use at lower } \\
\text { price }\end{array}$ \\
\hline 54 & Yondelis (Trabectedin) & Soft tissue sarcoma & Positive & $\begin{array}{l}\text { Rekomendacja prezesa AOTM } \\
\text { nr 19/2011 }\end{array}$ & $\begin{array}{l}\text { Major restriction: used restricted } \\
\text { to specific subpopulation. Minor } \\
\text { restriction: use at lower price }\end{array}$ \\
\hline 55 & Zavesca (Miglustat) & $\begin{array}{l}\text { Niemann-Pick type } \mathrm{C} \text { syndrome } \\
\text { (disease) }\end{array}$ & Positive & $\begin{array}{l}\text { Rekomendacja prezesa AOTM } \\
\text { nr 20/2011 }\end{array}$ & $\begin{array}{l}\text { Major restriction: resubmission } \\
\text { required after certain time } \\
\text { Minor restrictions: use at lower } \\
\text { price, monitoring required }\end{array}$ \\
\hline 56 & Xagrid (anagrelide) & $\begin{array}{l}\text { Indication classified to codes: } \\
\text { ICD-10: D. } 45 \text { with extensions } \\
\text { and D. } 47 \text { with extensions }\end{array}$ & Positive & $\begin{array}{l}\text { Rekomendacja prezesa AOTM } \\
\text { nr 142/2013 }\end{array}$ & Unrestricted \\
\hline 57 & Xagrid (anagrelide) & $\begin{array}{l}\text { Chronic myeloid leukemia (CML) } \\
\text { (ICD-10 C92.1) }\end{array}$ & Negative & $\begin{array}{l}\text { Rekomendacja prezesa AOTM } \\
\text { nr } 161 / 2013\end{array}$ & $\begin{array}{l}\text { Clinical reasons: insufficient } \\
\text { evidence for use (poor quality data) }\end{array}$ \\
\hline
\end{tabular}

than other attributes in half of the cases of pairwise comparisons (Table 3). The advancement in technology and manufacturing technology were found to be amongst the least impactful factors (Tables 3 and 4).

The sensitivity analysis indicated that six out of 13 criteria were deemed as equally impactful in the appraisal process (Table 2). The VIP minimum values and maximum regret of the remaining seven attributes did not pass the threshold test. The sensitivity results indicated that the criteria of "clinical evidence" could be considered as the key contributor to the decision making process of the HTA Appraisal Body. Its VIP maximum value was above the score for any other variable. In addition to that, the maximal regret of the "clinical evidence" equalled zero. Both VIP maximum values and maximum regret for safety aspects, costs of treatment, and availability of alternative therapies were set at 0.5. Consequently, they can be added to the list of the most important attributes of the recommendation process as well. With a VIP maximum value above 0.8 and a maximum regret of 0.512 , the criteria of "recommendations from other jurisdictions" can be also listed as a potentially impactful contributor to the decision making process.

The criteria of the "the advancement of technology" and "manufacturing costs" had their VIP minimum values set to zero. Both were associated with the maximal regret. Hence, they have the highest opportunity costs when listed among the key contributors to the HTA recommendation process.

Table 2 MCDA criteria, Test Anova, SLAM, AHP weights and the results of sensitivity analysis

\begin{tabular}{|c|c|c|c|c|c|c|}
\hline & Test Anova & SLAM & AHP weights & VIP min & VIP max & Max Regret \\
\hline Indication uniquenes & 0.8529 & 10 & $2.31 \%$ & 0.12 & 0.5 & 0.87 \\
\hline Disease rarity & 0.4815 & 8 & $3.34 \%$ & 0.3 & 0.66 & 0.69 \\
\hline Disease severity & 0.4837 & 9 & $3.38 \%$ & 0.23 & 0.5 & 0.76 \\
\hline Adv.tech. & 0.7574 & 12 & $0.42 \%$ & 0 & 0.05 & 1.00 \\
\hline Manufacturing technology & 0.2169 & 13 & $0.23 \%$ & 0 & 0.02 & 1.00 \\
\hline Therapeutic alternative & 0.3666 & 4 & $8.56 \%$ & 0.5 & 0.67 & 0.5 \\
\hline Sci. evid. Clin.eff & 0.2546 & 1 & $14.76 \%$ & 0.98 & 1.00 & 0.00 \\
\hline Benefits from use of medicine (safety aspects) & $0.0071^{*}$ & 3 & $12.68 \%$ & 0.5 & 0.81 & 0.5 \\
\hline Cost effectiveness analysis & 0.0747 & 5 & $9.86 \%$ & 0.48 & 1.00 & 0.52 \\
\hline Budget impact analysis & 0.646 & 6 & $10.26 \%$ & 0.44 & 0.58 & 0.56 \\
\hline Therapy cost & 0.2937 & 2 & $17.60 \%$ & 0.5 & 0.82 & 0.5 \\
\hline HTA recommendations issued elsewhere & 0.0691 & 7 & $11.88 \%$ & 0.48 & 0.83 & 0.51 \\
\hline Rationalization analysis & $0.0176^{*}$ & 11 & $4.73 \%$ & 0.13 & 0.5 & 0.87 \\
\hline
\end{tabular}

SLAM Simple Linear Additive Model, AHP Analytic Hierarchy Process, VIP Variable Interdependent Parameters, Sci. evid. clin.eff Scientific evidence for clinical efficiency, Adv.tech. Advancement of technology

*statistical significance at $p$-value $(\leq 0.05)$ 


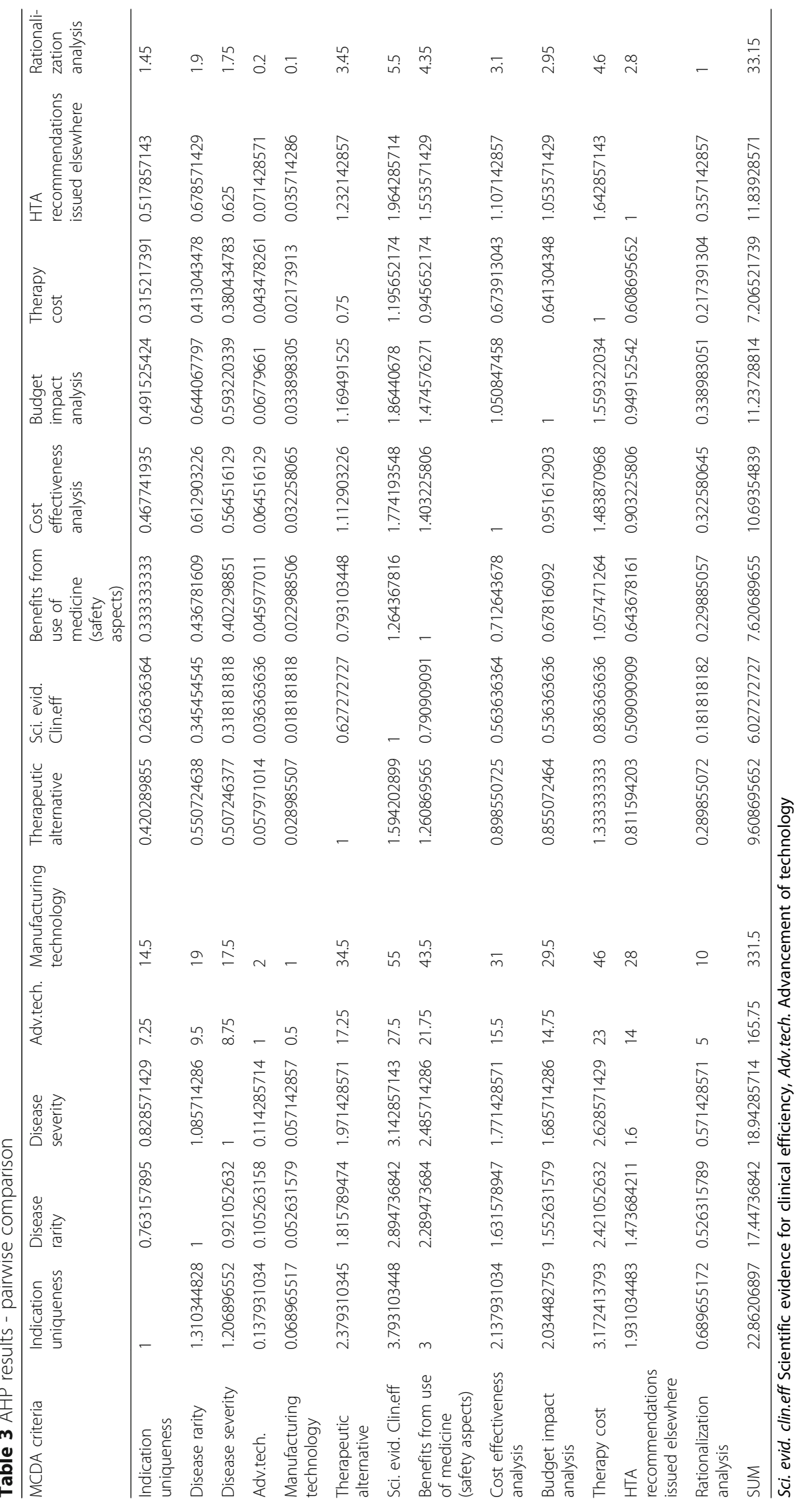




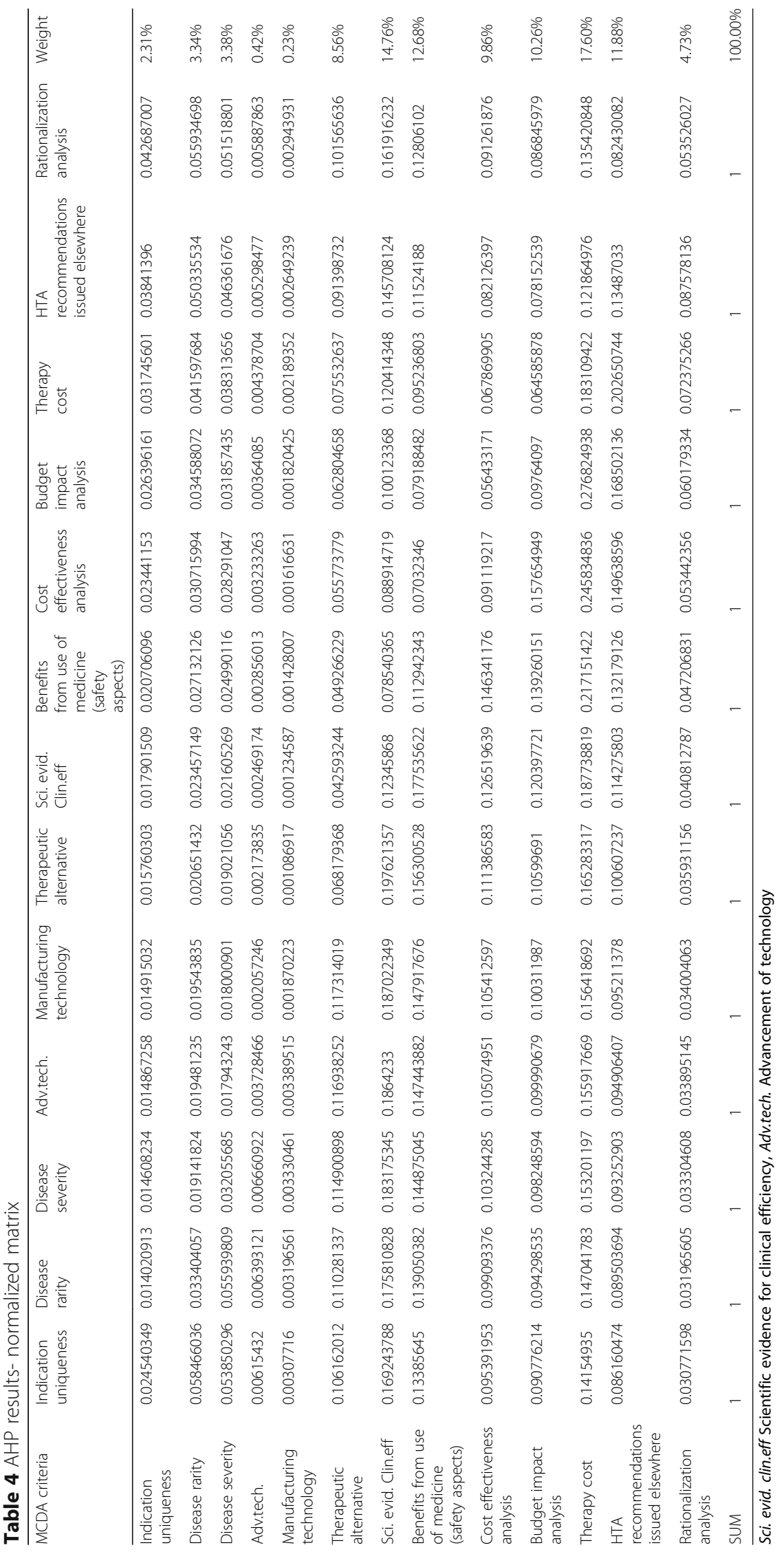


Taking the VIP results into consideration, there is a significant uncertainty with regards to the inclusion of "disease severity" and "disease rarity" among the decision-making criteria of the Polish HTA Appraisal Body as well. The same is true for "the results of rationalization analysis" and "indication uniqueness". While the minimum VIP value for all four criteria was below 0.5 , the maximal regret was above 0.5 .

\section{Discussion}

As the differences in access to OMPs across EU settings become more and more visible, there is a growing understanding that new approaches should be implemented to ensure a more transparent and fair allocation of funds across all individuals who suffer from rare diseases. Despite some international initiatives, new P\&R pathways tailored specifically to meet peculiarities of the value assessment of OMPs are still really scarce. The currently available HTA guidelines are mainly limited to the assessment of clinical and cost effectiveness as well as safety. In the field of rare diseases, the importance of the consideration of non-economic criteria in the P\&R decision-making is especially raised. Therefore, it was interesting to investigate what kind of value attributes are considered in the HTA process when there are no guidelines designed for the assessment of OMPs.

The study proved that both clinical evidence and economic considerations (CEA, BIA, the cost of therapy) played an important role in the assessment of OMPs in Poland. The results were consistent across different MCDA methods, and both the AHP weights and the SLAM rankings lead to similar conclusions.

The Polish Appraisal Body tended to rank the clinical evidence as the most important factor in the decisionmaking process. Interestingly, the notion of cost of therapy constituted the second largest contribution to the HTA recommendation process followed by the safety aspects. The importance of these two criteria, however, was similar, as could be seen in the comparison of AHP weights and the results of the sensitivity analysis. Nevertheless, it should be noted that there were statistically significant differences between positive and negative HTA recommendations in terms of the deliberation of safety aspects, which received more attention in the latter group.

According to both SLAM and AHP methods, the existence of a comparator was the fourth most impactful factor in the recommendation process at the Polish HTA agency. This was more important than disease rarity and severity. Interestingly, the results indicated that the availability of a therapeutic alternative was also valued more than economic evaluations. The weights of both CEA and BIA were set to $9 \%$. The sensitivity analysis provided more insight into the importance of economic evaluation. The maximum VIP results for the
CEA were set to one, which are only the second criteria with such a high value. if a lower regret is set with CEA versus $\mathrm{BIA}$, then the former was more influential in the recommendation process than the latter.

Even though the cost of therapy played such a significant role in the HTA appraisal process, the results of the budget impact analysis (BIA) did not contribute that heavily towards the HTA outcome. BIA's maximal regret set above 0.5 indicates that there is a significant opportunity cost for its inclusion in the list of recommendation making criteria.

Oddly enough, HTA recommendations issued by other HTA agencies also played an important role in the Appraisal Body's judgment. Their weight was almost as high as the one established for the economic criteria. In addition, it should be mentioned that the influence of other HTA agencies was greater for the negative rather than the positive HTA recommendations of the Polish Appraisal Body in a statistically significant manner.

Although the study produced some interesting results, it is not free from limitations, some of which are inherited within the MCDA methodology itself. Firstly, the consequences of the adaptation of the SLAM bring certain simplifications to the analysis that are not without an impact on the results. The SLAM assumes mutual preference independence, which means that preference for an outcome measured by one criterion is independent from the outcome related to another criterion. It can be envisaged, however, that the real value judgment of the HTA Appraisal Body may not be independent across different criteria in the same manner in which value attributes correlate with each other. SLAM introduces a complete compensatory rule that involves an offset mechanism where a bad performance on one criterion can be compensated by a good performance on others. In real life settings, it is, however, difficult to expect that the HTA Appraisal Body will be willing to trade the value of a given OMP between two different attributes. Secondly, the adaptation of AHP methods was applied without transforming the scale to Saaty's integer values 1-9. There are a number of approaches, which allow for this kind of switch with the adoption of the geometric scale [19], balanced scale [20], power [21], logarithmic [22], and finally root square [21]. It has to be underscored, however, that the analytic hierarchy process (AHP) method was selected following great consideration as it allows a pair-wise comparison of attributes. The application of AHP in this setting allowed the identification of intransitivity of collected criteria in the recommendation making process as well.

In addition to the limitations inherited in the MCDA methodology itself, there are also some additional risks of bias in our results related to the study design. Firstly, although we have taken into consideration all drug 
indication pairs assessed by the HTA agency between 2011 and 2015, we have not accounted for any potential changes in the Appraisal Body's preferences across the study period. Secondly, two reviewers subjectively conducted the assessment of attitudes towards different MCDA criteria. Thirdly, the study was limited to the HTA recommendations only. Some of the Appraisal Body's considerations failed to be presented in these documents, and the assessment of other P\&R decision makers were left outside of the scope of our analysis.

Despite these limitations, our study contributes to the growing body of literature focused on the adaptation of MCDA to the OMPs assessment. In contrast to other publications, it presents the application of MCDA to the analysis of preferences of decision makers towards the value attributes of OMPs. To our knowledge, none of previous studies had a similar objective. For instance, Schey et al. [23] adopted MCDA framework to the value OMPs from the UK perspective. Iskrov [24] developed an MCDA value model for the assessment of OMPs from the perspective of the Bulgarian payer. In the manuscript of Sussex J [25], the objective was to pilot the use of MCDA as a framework for a value assessment of OMPs.

Still, the current study could be regarded as a continuation of earlier research that verified the potential impact of the implementation of an MCDA approach on the value assessment of OMPs in the Polish context [14]. Despite different objectives and the scope of data analysed, there is some resemblance in the conclusions between both studies. Firstly, it was revealed that the economic arguments had an immense importance in the Polish HTA process. Secondly, it was indicated that other criteria beyond the set of value attributes defined in HTA guidelines, such as the cost of therapy, HTA outcomes from other jurisdictions, played a role in the appraisal process also in Poland.

In addition to MCDA studies, there are some publications that provide insight into the value attributes used by manufacturers in price setting for OMPs as well. Therefore, some comparisons can be made between the preferences of decision makers and producers. In this regard, at least three interesting observations should be noted. Firstly, the opposite valuation was found with respect to the criteria of the clinical effectiveness. Although the highest importance of data regarding efficacy in the recommendation process was clearly expressed, it has not been found among the predictors of the price of orphan drugs $[26,27]$. Secondly, some similarities could be drawn between the preferences of manufacturers and decision makers with respect to the availability of comparator. While it was valued as the fourth most relevant criteria in the HTA appraisal process, some studies indicated its importance in the manufacturers' setting of a pricing policy. For instance, the Belgian comparative analysis of prices of reimbursed OMPs revealed that total annual costs were lower for drugs with a comparator compared to those without alternative treatment options [28]. Finally, a contradiction could be noted with respect to the notion of disease rarity. As this was not listed amongst the key attributes of the HTA appraisal process, the opposite could be said with respect to pricing studies. This review of 75 OMPs, which were granted marketing authorization by the EMA until 2014, revealed a significant inverse relationship between disease prevalence and the annual cost of drug treatment [29]. However, analysis of 45 OMPs in the five biggest EU jurisdictions revealed that the number of available alternatives as well as the prevalence of disease was correlated with the price of the drug [30].

In order to ensure the contribution of our findings towards further improvements of access to treatments for rare diseases, some recommendations for future development of value framework of OMPs can be elicited. The first recommendation is dedicated to further enhancement of the broader engagement of different stakeholders' groups. Given the nature and peculiarities of rare diseases, uncertainty regarding both clinical and economic consequences of the implementation of a given health technology to the routine practice is inevitable. Therefore, it is not surprising that the Polish Appraisal Body attributed the highest value to the clinical evidence and noted the issue with credibility of data so many times among the reasons for negative HTA recommendation. To address such challenges, the development of a value framework should take views of both all decision makers and patient advocacy groups (PAGs) into consideration. With respect to the first group, the need of collaboration between the HTA agencies and EMA must be highlighted. Given the scarcity of clinical and safety data for OMPs, early dialog can be of great value especially when it comes to common understanding of unmet medical needs and scientific approaches to real life data collection. In addition to the wide range of incentives designed for OMPs available for the regular approval process, there are a number of special pathways for the marketing authorization such as accelerated, conditional, and exceptional pathways that enable the most needed OMPs to reach the patients in timely fashion [31]. Early engagement of HTA bodies in such processes could potentially facilitate a generation of P\&R outcomes as well. As far as the contribution of the second group is concerned, it is again the lack of sufficient clinical and safety data that makes the PAG's role in the P\&R decision making so valuable. Given limited evidence regarding particular health states, patient experience can provide a needed insight into the real burden of disease. Such individual stories cannot compete with the breadth of evidence available for common diseases. Nevertheless, as long as it is ensured that patients' stories are collected in a systematic and transparent manner, it can certainly successfully address some of the uncertainties of the HTA process. The second 
recommendation calls for the introduction of new innovative pricing \& reimbursement arrangements. The results of our study indicated that the notion of the cost of therapy was a key consideration during the recommendation process of OMPs. At the same time, the price of a drug and an unfavorable cost effectiveness ratio were the most frequent reasons for the negative HTA recommendations. Therefore, a new set of P\&R rules should enable the implementation of a special form of innovative managed entry agreements. Alternatively, a financing mechanism that links reimbursement with drug performance can be introduced to validate cost-effectiveness claims. Following the preferences of expert payers, the second one should especially be taken into consideration in the CEE Region [32]. Temporary access to OMPs could be secured as long as an HTA agency envisages the opportunity for further real life data collection before a final recommendation has been completed. For example, this is a common approach in the Netherlands and Sweden where temporary coverage is granted conditional upon the conduct of an observational study.

\section{Conclusions}

In summary, it can be concluded that our study provides an interesting insight into the value judgment of the Polish HTA process. It also indicates that the decision-making preference of the HTA Appraisal Body goes beyond a standard set of HTA criteria. In addition to clinical and economic evidence, such criteria as the cost of therapy and the rationalization analysis, along with HTA recommendations from other HTA agencies, played a significant role without being included in the HTA guidelines. Therefore, there is a need for a new approach to the value assessment of OMPs that will even take into consideration those criteria that are not yet formally included in the HTA framework. Unless this happens, transparency and objectivity of the decision-making process cannot be fully ensured, and equity with regard to access to OMPs cannot be improved.

\section{Additional file}

Additional file 1: MCDA analysis. (DOCX $9 \mathrm{~kb}$ )

\footnotetext{
Abbreviations

AHP: Analytic hierarchy process; AHTAPoL: Polish health technology assessment agency; BIA: Budget impact analysis; CEE: Central and Eastern European; EMA: European medicines agency; EU: European Union; HTA: Health technology assessment; ISPOR: International Society for pharmacoeconomics and outcomes research; LSDP: Life saving drug program; MAVT: Multiple attribute value theory; MoCA: Mechanism for coordinated access; OMPs: Orphan medicinal products; OV: Overall value; P\&R: Pricing and reimbursement; SLAM: Simple linear additive model; TVF: Transparent value framework; VIP: Variable interdependent parameters
}

\section{Acknowledgements}

KK drafted the manuscript, collected raw data and supervised the analysis of the data. VZ analysed the data and prepared jointly with KK and ZK the manuscript. $\mathrm{TL}$ analysed the data. KMZ collected raw data and contributed to the analysis. ZK designed the study and prepared jointly with KK and ZK the manuscript.

Authors would like to thank ZRx Outcomes Research Inc. for the utilization of ZRx MCDA tool.

Funding

Neither sponsorship nor grants have been received for the conducted study.

Availability of data and materials

Data sharing not applicable to this article as no datasets were generated or analysed during the current study.

\section{Authors' contributions}

All authors contributed equally to this work. All authors read and approved the final manuscript.

Ethics approval and consent to participate

Not applicable.

\section{Competing interests}

The authors declare that they have no competing interests.

\section{Publisher's Note}

Springer Nature remains neutral with regard to jurisdictional claims in published maps and institutional affiliations.

\section{Author details}

${ }^{1}$ Department of Health Economics and Healthcare Management, Kozminski University, Warsaw, Poland. ${ }^{2}$ Advanced Management Training Programme in Pharmacoeconomics, Pharmaceutical Marketing and Law, Warsaw University of Technology, Warsaw, Poland. ${ }^{3} \mathrm{HEOR}$, ZRx Outcomes Research, Toronto, Canada. ${ }^{4}$ Department of Health Policy \& Health Economics, Faculty of Social Sciences, Eötvös Loránd University, Budapest, Hungary. ${ }^{5}$ Department of Econometrics, Poznan University of Economics, Poznan, Poland. ${ }^{6}$ Syreon Research Institute, Poznan University of Economics, Budapest, Hungary.

Received: 13 August 2017 Accepted: 10 April 2018

Published online: 27 April 2018

\section{References}

1. Tejada P. Survey: patients' access to orphan drugs in Europe. Paris: The EURORDIS Rare Disease Europe; 2009. http://www.eurordis.org/content/ survey-patients'-access-orphan-drugs-europe. Accessed 26 Jan 2016

2. Process on Corporate Social Responsibility in the Field of Pharmaceuticals Platform on Access to Medicines in Europe Working Group on Mechanism of Coordinated Access to Orphan Medicinal Products (MoCA-OMP) Ref. Ares (2014) 3857065-19/11/2014. European Union. 2014. http://ec.europa.eu/ DocsRoom/documents/7629/attachments/1/translations/en/renditions/pdf. Accessed 26 Jan 2016

3. Gutierrez L, Patris J, Hutchings A, Cowell W. Principles for consistent value assessment and sustainable funding of orphan drugs in Europe. Orphanet J Rare Dis. 2015;10:53.

4. National Institute for Health and Care Excellence. Interim process and methods of the highly specialised technologies Programme. London: National Institute for Health and Care Excellence; 2016. http://www.nice.org. uk/Media/Default/About/what-we-do/NICE-guidance/NICE-highlyspecialised-technologies-guidance/Highly-Specialised-Technologies-Interimmethods-and-process-statements.pdf. Accessed 20 Jan 2016

5. Life Saving Drugs Program Criteria and Conditions. Australian Government: The Departament of Health; 2013. http://www.health.gov.au/internet/main/ publishing.nsf/Content/Isdp-criteria. Accessed 15 Jan 2016.

6. Skoupa J, Annemans L, Hájek P. Health economic data requirements and availability in the European Union: results of a survey among 10 European countries. Value In Health Regional Issues. 2014;4(C):53-7.

7. The Pharmaceutical Law and the Act on the Reimbursement of Medicines, Foodstuffs Intended for Particular Nutritional Uses and Medical Devices in Poland. J Laws. 2011; No., 122, item 696. http://prawo.sejm.gov.pl/isap.nsf/ 
DocDetails.xsp?id=WDU20111220696. Acessed 22 Apr 2018.10.1186/s13023018-0803-9

8. Iskrov G, Miteva-Katrandzhieva T, Stefanov R. Challenges to orphan drugs access in Eastern Europe: the case of Bulgaria. Health Policy. 2012;108(1):10-8.

9. Logviss K, Krievins D, Purvina S. Rare diseases and orphan drugs: Latvian story. Orphanet J Rare Dis. 2014:9:147.

10. Le Cam Y. Inventory of access and prices of orphan drugs across Europe: a collaborative work between national alliances on rare diseases \& EURORDIS. Presentation, Paris 2010. EURORDIS Rare Disease Europe. 2016. http://img. eurordis.org/newsletter/pdf/mar-2011/ERTC_13122010_YLeCam_Final.pdf. Accessed 20 Jan 2016.

11. Aotm.gov.pl. Warsaw: The Agency for Health Technology Assessment and Tariff System. 2016. http://www.aotm.gov.pl/www/hta/rada-przejrzystosci. Accessed 01 Aug 2017.

12. Diaby V, Goeree R. How to use multi-criteria decision analysis methods for reimbursement decision-making in healthcare: a step-by-step guide. Expert Rev Pharmacoecon Outcomes Res. 2014;14(1):81-99.

13. Zah $\mathrm{V}$, Thompson S, Berjan M, Goeree R. Zrx Mcdm: a fully flexible tool to support the local adaptation of multiple-criteria decision criteria in health care. Value Health. 2015;18(7):A703.

14. Kolasa K, Zwolinski KM, Kalo Z, Hermanowski T. Potential impact of the implementation of multiple-criteria decision analysis (MCDA) into the polish pricing and reimbursement process of orphan drugs. Orphanet $」$ Rare Dis. 2016;11:23.

15. Marsh K, IJzerman M, Thokala P, Baltussen R, Boysen M, Kaló Z, Lönngren T, Mussen F, Peacock S, Watkins J, Devlin N. Multiple criteria decision analysis for health care decision making-emerging good practices: report 2 of the ISPOR MCDA emerging good practices task force. Value Health. 2016;19(2):125-37.

16. Kolasa K, Wasiak R. Health technology assessment in Poland and Scotland: comparison of process and decisions. Int J Technol Assess Health Care. 2012:28(1):70-6.

17. Thokaka P, Devlin N, Marsh K, Baltussen R, Boysen M, Kalo Z, et al. Multiple criteria decision analysis for health care decision making — an introduction: report 1 of the ISPOR MCDA emerging good practices task force. Value Health. 2016:19:1-13.

18. van Herwijnen M. Multiple?Attribute value theory (MAVT). Amsterdam: Institute for Environmental Studies (IVM); 2016. http://www.ivm.vu.nl/en/ Images/MCA1_tcm234-161527.pdf. Accessed 15 Jan 2016

19. Lootsma FA. Theory and methodology conflict resolution via pairwise comparison of concessions. Eur J Oper Res. 1989;40:109-16.

20. Salo A, Hamalainen R. On the measurement of preference in the analytic hierarchy process. J Multicrit Decis Anal. 1997;6:309-19.

21. Harker PT, Vargas LG. The theory of ratio scale estimation: Saaty's analytic hierarchy process. Manag Sci. 1987;33(11):1383-403.

22. Ishizaka A, Balkenborg D, Kaplan T. Influence of aggregation and measurement scale on ranking a compromise alternative in AHP. J Oper Res Soc. 2011;62(4):700-10.

23. Schey C, Krabbe PFM, Postma MJ, Connolly MP. Multi-criteria decision analysis (MCDA): testing a proposed MCDA framework for orphan drugs. Orphanet J Rare Dis. 2017;12(1):10.

24. Iskrov G, Miteva-Katrandzhieva T, Stefanov R. Multi-criteria decision analysis for assessment and appraisal of orphan drugs. Front Public Health. 2016:4:214.

25. Sussex J, Rollet P, Garau M, Schmitt C, Kent A, Hutchings A. A pilot study of multicriteria decision analysis for valuing orphan medicines. Value Health. 2013;16(8):1163-9.

26. Kanters TA, de Sonneville-Koedoot C, Redekop WK, Hakkaart L. Systematic review of available evidence on 11 high-priced inpatient orphan drugs. Orphanet J Rare Dis. 2013;8:124.

27. Joppi R, Bertele' V, Garattini S. Orphan drugs, orphan diseases. The first decade of orphan drug legislation in the EU. Eur J Clin Pharmacol. 2013; 69(4):1009-24.

28. Simoens S. Pricing and reimbursement of orphan drugs: the need for more transparency. Orphanet J Rare Dis. 2011;6:42.

29. Onakpoya IJ, Spencer EA, Thompson MJ, Heneghan CJ. Effectiveness, safety and costs of orphan drugs: an evidence-based review. BMJ Open. 2015:5:e007199.
30. Aballéa S, Toumi M, Vataire AL, Millier A, Lamure M. PHP6 quantitative analysis of the influence of disease and product characteristics on orphan drug prices in Europe. Value Health. 2010;13(3):A82.

31. Ogbah R. Orphan medicinal products - a European process overview. Regulatory Rapporteur. 2015;12(2):5-11.

32. Kolasa K, Kalo Z, Hornby E. Pricing and reimbursement frameworks in Central Eastern Europe: decision tool to support choices. Expert Rev Pharmacoecon Outcomes Res. 2015;15(1):145-55.

\section{Ready to submit your research? Choose BMC and benefit from:}

- fast, convenient online submission

- thorough peer review by experienced researchers in your field

- rapid publication on acceptance

- support for research data, including large and complex data types

- gold Open Access which fosters wider collaboration and increased citations

- maximum visibility for your research: over $100 \mathrm{M}$ website views per year

At BMC, research is always in progress.

Learn more biomedcentral.com/submissions 